\title{
Ambipolar Diffusion Effects on Weakly Ionized Turbulence Molecular Clouds
}

\author{
Pak Shing $\mathbf{L i}^{1}$, Christopher F. McKee ${ }^{2}$, and Richard I. Klein ${ }^{3}$ \\ ${ }^{1}$ Astronomy Department, University of California, Berkeley, CA 94720, USA \\ email: psli@astro.berkeley.edu \\ ${ }^{2}$ Physics Department and Astronomy Department, University of California, \\ Berkeley, CA 94720, USA \\ email: cmckee@astro.berkeley.edu \\ ${ }^{3}$ Astronomy Department, University of California, Berkeley, CA 94720, USA; \\ and Lawrence Livermore National Laboratory, P.O.Box 808, L-23, Livermore, CA 94550, USA \\ email: klein@astron. berkeley.edu
}

\begin{abstract}
Ambipolar diffusion (AD) is a key process in molecular clouds (MCs). Non-ideal MHD turbulence simulations are technically very challenging because of the large Alfvén speed of ions in weakly ionized clouds. Using the Heavy-Ion Approximation method (Li, McKee \& Klein 2006), we have carried out two-fluid simulations of AD in isothermal, turbulent boxes at a resolution of $512^{3}$, to investigate the effect of $\mathrm{AD}$ on the weakly ionized turbulence in MCs. Our simulation results show that the neutral gas component of the two-fluid system gradually transforms from an ideal MHD turbulence system to near a pure hydrodynamic turbulence system within the standard $\mathrm{AD}$ regime, in which the neutrals and ions are coupled over a flow time. The change of the turbulent state has a profound effect on the weakly ionized MCs.
\end{abstract}

Keywords. turbulence, ISM: magnetic fields, ISM: clouds, methods: numerical.

\section{Introduction}

For an ideal MHD fluid, the magnetic field is assumed to be frozen into the ionized gas. From observations, the ionization fraction of dense molecular clouds (MCs) is very low, on the order of $10^{-7}$ or less. Here we treat the MC with a highly simplified 2-fluid model composed of only neutral and ionized molecules. The gyro-frequency of an ion is $\omega_{\mathrm{ci}}=e B / m_{i} c=9.58 \times 10^{-9} Z B m_{p} / m_{i} \mathrm{rad} \mathrm{s}^{-1}$, which is still very large compared to the ion-neutral collision frequency, $t_{\text {in }}^{-1}=\gamma_{\mathrm{AD}} \rho_{n} \sim 10^{-6} \mathrm{~s}$ inside typical MCs. At the MC densities, the ions are tied to the magnetic field. The neutral gas feels the presence of the magnetic field indirectly through collisions with the ions. With such a low ionization fraction, treating the weakly ionized MCs using ideal MHD is inappropriate because the coupling between ions and neutrals is weak. The result is that the majority of the MC gas will slowly drift relative to the magnetic field. This slow ambipolar diffusion (AD) process is important because it allows gravitational collapse even in the cloud that is initially magnetically sub-critical.

However, many observations show that MCs are in a supersonically turbulent state. If the $\mathrm{AD}$ time scale is much longer than the characteristic time scale of the turbulence, $\mathrm{AD}$ is unimportant. The observed line-width size relation implies that the dynamical time in a dense clump with size $\lesssim 1 \mathrm{pc}$ is comparable to the $\mathrm{AD}$ time scale. Can we ignore $\mathrm{AD}$ and use ideal MHD to treat these small regions in MCs? We use non-ideal MHD numerical simulations with $\mathrm{AD}$ to answer this question. 


\section{Numerical Method and Simulation Model Parameters}

We have implemented a two-fluid semi-implicit method into ZEUS-MP code using Mac Low \& Smith(1997) approach. We assume that the MCs are isothermal. As a result, the MHD equations for the two fluids, ions and neutrals, with $\mathrm{AD}$ are:

$$
\begin{aligned}
\frac{\partial \rho_{n}}{\partial t} & =-\nabla \cdot\left(\rho_{n} \mathbf{v}_{n}\right), \\
\frac{\partial \rho_{i}}{\partial t} & =-\nabla \cdot\left(\rho_{i} \mathbf{v}_{i}\right), \\
\rho_{n} \frac{\partial \mathbf{v}_{n}}{\partial t} & =-\rho_{n}\left(\mathbf{v}_{n} \cdot \nabla\right) \mathbf{v}_{n}-\nabla P_{n}-\gamma_{\mathrm{AD}} \rho_{i} \rho_{n}\left(\mathbf{v}_{n}-\mathbf{v}_{i}\right), \\
\rho_{i} \frac{\partial \mathbf{v}_{i}}{\partial t} & =-\rho_{i}\left(\mathbf{v}_{i} \cdot \nabla\right) \mathbf{v}_{i}-\nabla P_{i}-\gamma_{\mathrm{AD}} \rho_{i} \rho_{n}\left(\mathbf{v}_{i}-\mathbf{v}_{n}\right)+\frac{1}{4 \pi}(\nabla \times \mathbf{B}) \times \mathbf{B}, \\
\frac{\partial \mathbf{B}}{\partial t} & =\nabla \times\left(\mathbf{v}_{i} \times \mathbf{B}\right), \\
\nabla \cdot \mathbf{B} & =0
\end{aligned}
$$

where $\rho$ is the density, $\mathbf{v}$ is the velocity, $\mathbf{B}$ is the magnetic field strength, and $\gamma_{\mathrm{AD}}$ is the ion-neutral collisional coupling constant. The subscripts $i$ and $n$ denote ions and neutrals, respectively. Note that our simulations do not include gravity because we want to understand the complex interaction between $\mathrm{AD}$ and turbulence first. The two momentum equations with a stiff drag term are solved implicitly. Using this approach, the time step size is proportional to the grid size $\Delta x$ (Mac Low \& Smith 1997).

We use the Heavy-Ion Approximation (Li, McKee \& Klein 2006) to speed up the computation by a substantial factor. The principle of the Heavy-Ion Approximation (HIA) is to increase the mass of the ions by a large factor in order to reduce the frequency of ion Alfven waves so that the numerical time step size can be significantly increased. At the same time, $\gamma_{\mathrm{AD}}$ is reduced by the same amount to keep the drag force term the same. In Li, McKee \& Klein (2006), we show that there is a numerical criterion when using HIA: the inertial force term for the ions must be much smaller than the Lorentz force or the drag force term in the ion momentum equation. That leads to the condition for the accuracy of the approximation:

$$
R_{\mathrm{AD}}\left(\ell_{v_{i}}\right) \gg \mathcal{M}_{\mathrm{A} i}{ }^{2}
$$

where $\ell_{v_{i}}$ is the velocity length scale, $\mathcal{M}_{\mathrm{A} i}$ is the ion Alfvén Mach number, and $R_{\mathrm{AD}}$ is the AD Reynolds number,

$$
R_{\mathrm{AD}}(\ell) \equiv \frac{\ell v}{t_{\mathrm{ni}} v_{A}^{2}}=\frac{4 \pi \gamma_{\mathrm{AD}} \rho_{i} \rho_{n} \ell v}{\left\langle B^{2}\right\rangle}=\frac{\ell}{\ell_{\mathrm{AD}}} ;
$$

where $\ell_{\mathrm{AD}}$ is the $\mathrm{AD}$ length scale. $R_{\mathrm{AD}}$ is found to be a good parameter to measure the coupling strength between the ions and the neutral gas $(\mathrm{Li}, \mathrm{McKee} \&$ Klein 2006, Li et al. 2008). When $R_{\mathrm{AD}} \gg 1$, coupling is strong, and when $R_{\mathrm{AD}} \lesssim 1$, coupling is weak.

We have five $512^{3} \mathrm{AD}$ models driven at Mach 3 at the largest scale, $k=\ell_{0} / \lambda=1 \sim 2$, where $\ell_{0}$ is the box length. If the line-width size relation is satisfied, the size of the box is $\sim 0.41 \mathrm{pc}$. The simulation box is periodic and the temperature is at $10 \mathrm{~K}$. The plasma $\beta=0.1$ so that the magnetic field is strong. All simulations ran for 3 crossing time. $R_{\mathrm{AD}}\left(\ell_{0}\right)$ spans 4 orders of magnitude in these models, from 0.12 to 1200 , enough to cover the range from weak to strong AD. We also have an ideal MHD and a pure hydrodynamic run to compare with the strong and weak AD coupling cases. 


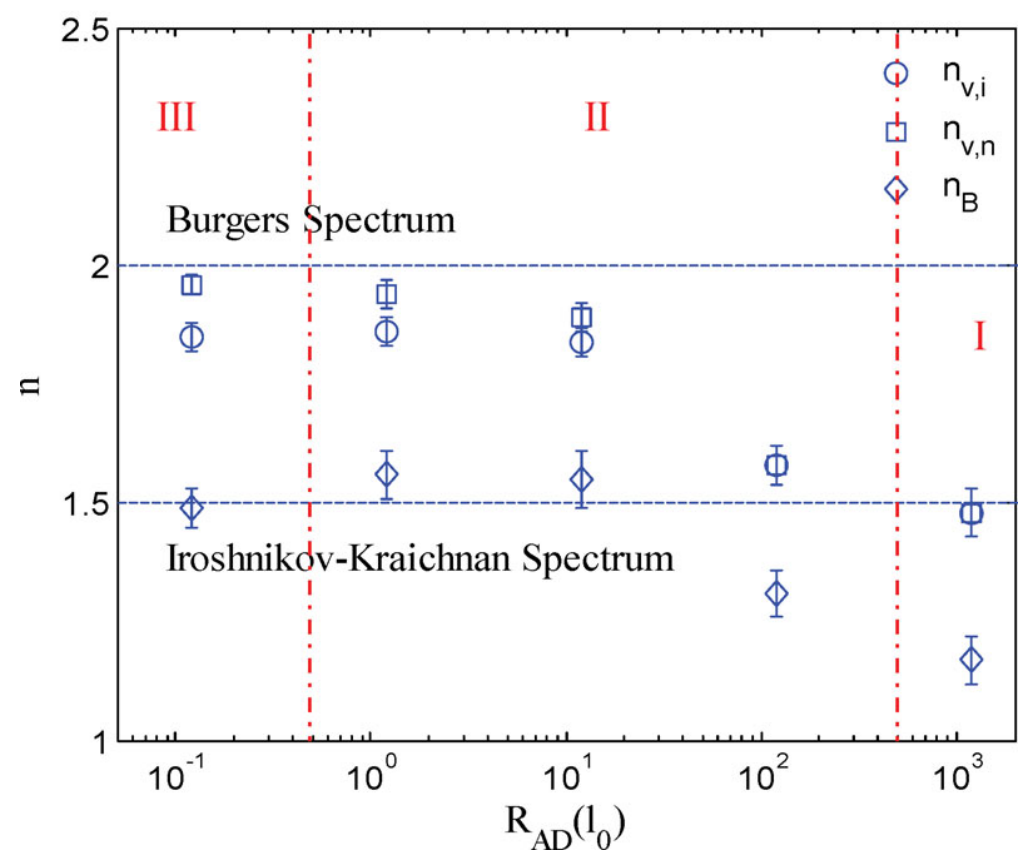

Figure 1. Change of the velocity and magnetic field power spectral indexes of ions and neutrals $\left(n_{v i}, n_{v n}\right.$, and $\left.n_{B}\right)$ as functions of $R_{\mathrm{AD}}$. The transition from the ideal MHD regime (I) to the pure hydro regime begins in the standard AD regime (II). The strong AD regime (III) is also shown. The ion velocity spectral index diverges from the neutral velocity spectral index when AD becomes strong.

\section{Simulation Results: Velocity Power Spectrum}

We present only the AD effect on the velocity power spectrum of a weakly ionized MC in this short report. For the low Alfvén Mach number in our simulations, the 1D velocity power spectrum of ideal MHD turbulence will be an Iroshnikov-Kraichnan spectrum, with an index of $3 / 2$. For a pure hydrodynamic system, the velocity spectral index is 2 , a shock dominated Burgers spectrum. In Figure 1, we plot the ion and neutral velocity spectral indexes and the magnetic field spectral indexes versus $R_{\mathrm{AD}}$ for the five turbulent $\mathrm{AD}$ models. The neutral velocity spectral indexes of our five AD models trace a transition between the ideal MHD and the pure hydrodynamic regimes. We can categorize five different regimes for $\mathrm{AD}$ (McKee, Li, \& Klein 2010) by comparing $R_{\mathrm{AD}}$ to the ion and neutral Alfvén Mach numbers. Figure 1 shows only the ideal MHD (regime I: $R_{\mathrm{AD}} \rightarrow \infty$ ), standard AD (regime II: $R_{\mathrm{AD}}>\mathcal{M}_{\mathrm{A}}$ ), and strong AD (regime III: $\mathcal{M}_{\mathrm{A}}>R_{\mathrm{AD}}>\mathcal{M}_{\mathrm{A} i}$ ) regimes. The change in the spectral index occurs mainly inside the standard AD regime, where the neutrals and ions are coupled together over a dynamic time scale and the damping of Alfvén waves of wavelength comparable to the size of the region is weak. Note that the ion power spectral index diverges from the neutral index when the coupling becomes weak. The magnetic field spectral indexes show a similar behavior.

Future observations could verify the change of the velocity power spectral index predicted by our simulations. Crutcher (1999) reports 27 molecular clumps with sensitive magnetic field measurements. Using the cloud clump diameter as the length scale and other measured parameters, we computed $R_{\mathrm{AD}}$ for these 27 clouds as shown in Figure 2 (unshaded histogram), including the 12 clouds with only upper limits on the magnetic 


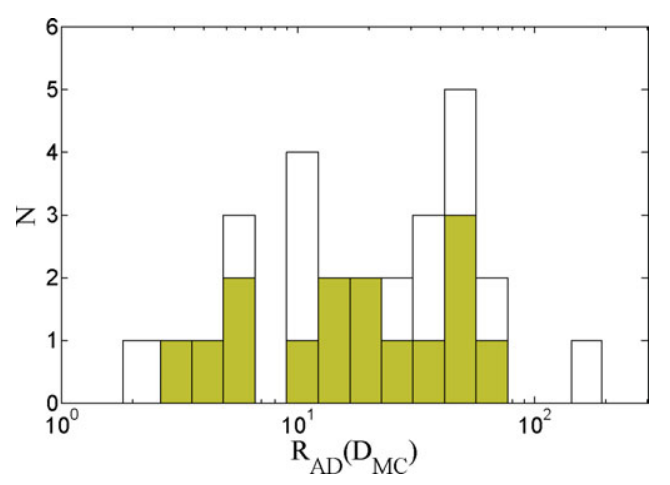

Figure 2. Histograms of the AD Reynolds numbers of $27 \mathrm{MC}$ clumps observed by Crutcher (1999). The shaded histogram shows only the 15 clumps with detected magnetic field strength.

field strength. The logarithmic mean of $R_{\mathrm{AD}}$ of the 15 clouds with detected magnetic fields (shaded histogram) is 17 (McKee, Li, \& Klein 2010). All these clouds have $R_{\mathrm{AD}}$ inside the standard AD regime. Once the velocity spectral indexes of these clouds are determined, we shall be able to determine if the spectral indexes of these clouds change with $R_{\mathrm{AD}}$ as predicted.

\section{Conclusion}

We report our non-ideal MHD turbulence simulations of the effects of AD on weakly ionized MCs. We found that the neutral velocity spectral index in a strong magnetic field environment changes from the Iroshnikov-Kraichnan spectrum expected for a strong Bfield system to a Burgers spectrum when $\mathrm{AD}$ is strong, as the result of the decoupling of neutrals from ions. Other effects of AD on weakly ionized MCs, such as the clump mass function, the mass-flux ratio, and the line-width size relation are reported in Li et al. (2008), McKee, Li \& Klein (2010), Li, McKee \& Klein (2010). Our simulations show that $\mathrm{AD}$ is important in changing the turbulent state of weakly ionized MCs.

\section{Acknowledgements}

This research has been supported by NASA under an ATFP grant NNX09AK31G, the NSF under grant AST-0908553 (CFM and RIK), and the high performance computing resources from the NCSA through the grant TG-MCA00N020. CFM also acknowledges the support of the Groupement d'Intérêt Scientifique (GIS) "Physique des deux infinis (P2I)." RIK received support for this work provided by the US Department of Energy at Lawrence Livermore National Laboratory under contract DE-AC52-07NA 27344.

\section{References}

Crutcher, R. M. 1999, ApJ, 520, 706

Li, P. S., McKee, C. F., \& Klein, R. I. 2006, ApJ, 653, 1280

Li, P. S., McKee, C. F., Klein, R. I., \& Fisher, R. T. 2008, ApJ, 684, 380

Li, P. S., McKee, C. F., \& Klein, R. I. 2010, in preparation

McKee, C. F., Li, P. S., \& Klein, R. I. 2010, ApJ, accepted, arXiv:1007.2032

Mac Low, M.-M. \& Smith, M. D. 1997, ApJ, 491, 596 